
June 1939

\title{
DEVELOPMENT OF A FIBROUS TEXTURE IN COLD- WORKED RODS OF COPPER
}

\author{
By Herbert C. Vacher
}

\begin{abstract}
Mono- and bicrystalline rods of copper were subjected to different degrees of cold-swaging and were examined with $\mathrm{X}$-ray diffraction and metallographic methods. The results indicate that swaging causes the crystals to rotate until either an octahedral or a cubic axis becomes parallel with the rod axis. Whether the crystal assumes the octahedral or the cubic orientation depends upon which axis initially formed the smaller angle with the rod axis. Crystals approaching the octahedral orientation developed secondary crystals, after approximately 60 -percent reduction in area. On further swaging, these secondary crystals approached the cubic orientation. Crystals initially approaching the cubic orientation remained one crystal after 95-percent reduction in area.
\end{abstract}

\section{CONTENTS}

I. Introduction

II. Procedures and results. 652

1. Material and swaging

2. Changes in orientation resulting from swaging

3. Appearance of the ends of swaged rods _._.

4. Metallographic examination

5. Orientations of secondary crystals_._.

III. Discussion

1. Manner of changing the orientation of a copper crystal by swaging

2. Bending of slip planes and a possible origin of secondary crystals_ 664

3. Fibrous texture of cold-worked rods.

IV. Summary _ . 667

V. References

\section{INTRODUCTION}

X-ray diffraction patterns indicate that a severely cold-worked metal consists of small crystal fragments called crystallites. The ultimate size of the crystallite is of the order of $10^{-5} \mathrm{~cm}$ and is independent of the cold-working process. However, if the metal is a rod whose diameter has been reduced more than about 30 percent by cold-working, the crystallites are found to be in a position where a particular crystallographic axis is more or less parallel to the rod axis. Other axes of the crystallites are randomly oriented in respect to a direction perpendicular to the rod axis. This type of structure is referred to usually as a fibrous texture and is developed from crystals that initially were randomly oriented. The particular axis, however, that is parallel to the rod axis is not always the same for different metals, for example, in rods of iron or aluminum a dodecahedral axis 
and an octahedral axis, respectively, are parallel to the rod axis. Some metals, such as copper, gold, and silver, produce a fibrous texture in which some crystallites have an octahedral axis and others have a cubic axis parallel to the rod axis.

The microstructure of a cold-worked rod shows that crystals initially equiaxed are elongated in the direction of the rod axis and resemble thread-like fibers. The structure within the individual fiber is homogeneous as far as can be ascertained from the literature, that is, the banded structure frequently observed in the crystals of metals cold-worked by flat rolling evidently is not common in round rods. Therefore, it appears that a cold-worked rod consists of fibers which are composed of small crystallites having a preferred orientation.

In copper rods where the fibrous texture consists of two orientations, an octahedral axis or a cubic axis parallel to the rod axis, each fiber must represent one of these orientations. There has been no experimental work to verify this conclusion or to indicate how these apparently stable orientations are developed. In order to supply this information, it is necessary to show the history of the changes in structure occurring when crystals with different orientations are elongated into fibers. This has been done by swaging large crystals of copper and determining the structure after different degrees of cold-work with the usual metallographic and X-ray-diffraction methods.

\section{PROCEDURES AND RESULTS}

\section{MATERIAL AND SWAGING}

Electrolytic copper of wire-bar purity was melted in a graphite crucible and with an Arsem furnace. The crucible was shaped to form rods $3 / 8$ in. in diameter and 6 in. long. This procedure produced oxygen-free rods of copper which were then converted by the Bridgman [1] ${ }^{1}$ method into rods consisting of one or a few large crystals. To do this, several of the rods were placed in close-fitting holes drilled in a cylinder of graphite and then lowered slowly (about 4 in. per hour) through a furnace maintained at approximately $1,100^{\circ} \mathrm{C}$. The resulting rods were 0.337 in. in diameter and usually consisted of from one to four crystals running approximately parallel to the rod axis. Four rods were used in this investigation, two rods each consisting of one crystal and two rods each consisting of two crystals. Both crystals of each bicrystalline rod extended the full length of the rod, so that a cross section always showed two crystals.

Each of the rods was cold-swaged progressively through 19 tapered dies to a reduction in area of approximately 95 percent. Specimens, representing reductions in area of approximately $30,60,80$, and 95 percent, were cut from the rods and used for examination with the microscopic and X-ray-diffraction methods. Table 1 shows the various steps that were employed in reaching each of the above stages in reduction.

1 Figures 1 to 9 in brackets indicate the literature references at the end of this paper. 


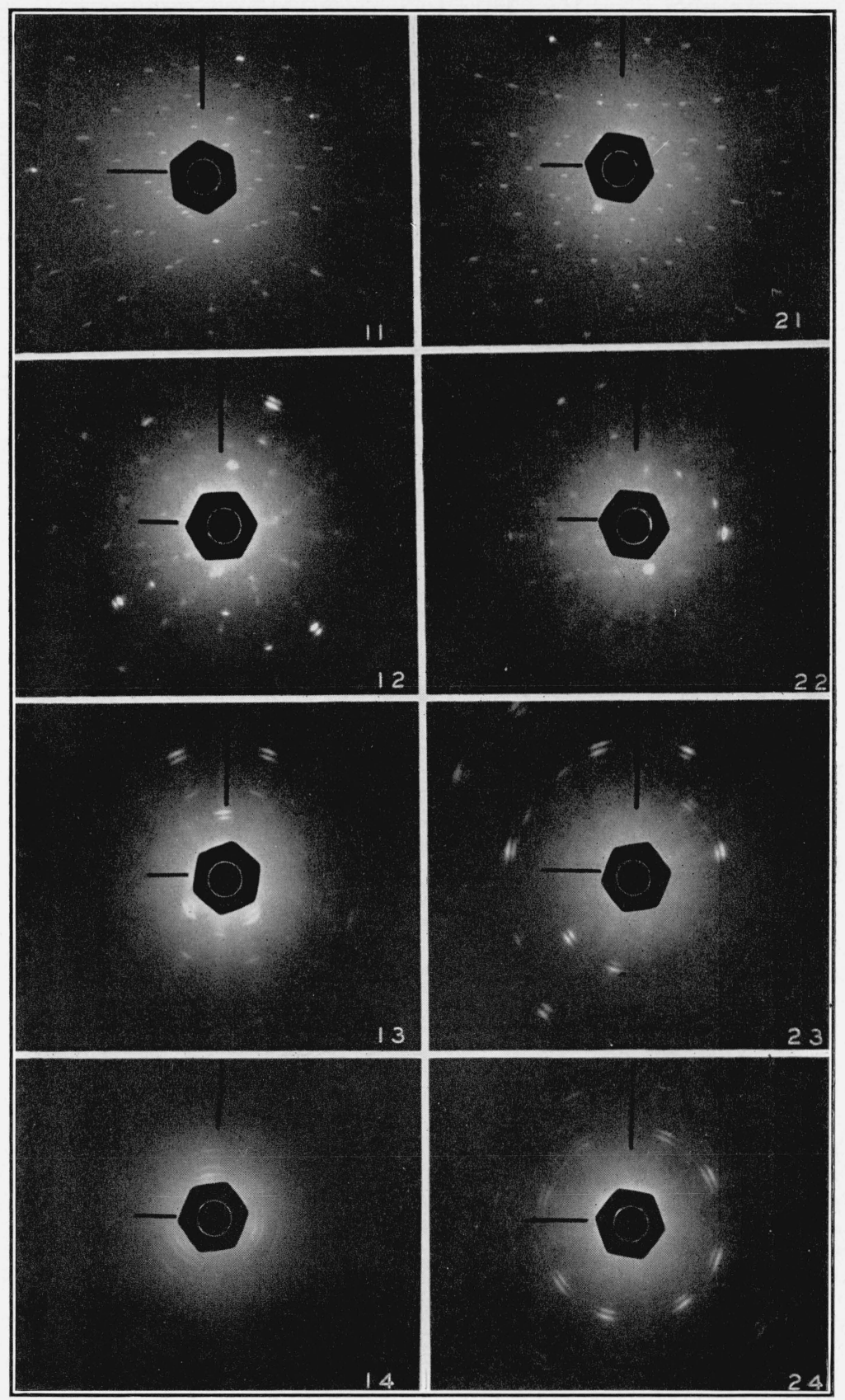

FigURE 1.- "Back-reflection" diffraction patterns showing the changes in orientation of monocrystalline rods 1 and 2 after increasing degrees of swaging.

The first and second digits of the number in the lower right corner of the patterns are the rod number and the identification number shown in table 1, respectively. Unfiltered molybdenum radiation; $28 \mathrm{kvp}$; 1- by 6 -cm pinhole; $3.0-\mathrm{cm}$ film-to-specimen distance. 
TABLE 1.-Dies used in obtaining different degrees of swaging

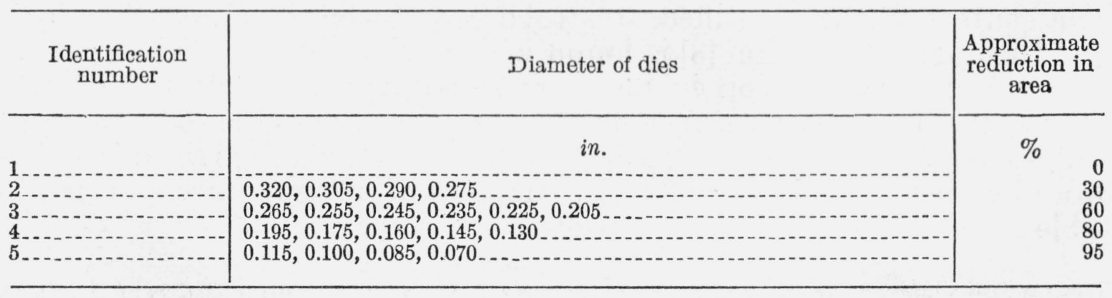

\section{CHANGES IN ORIENTATION RESULTING FROM SWAGING}

Patterns indicating diffractions from an area near the center of a crystal, shown in cross sections of the rods, were made by the backreflection method after deeply etching the rods with diluted nitric acid to remove any effects resulting from their preparation. In making these patterns the collimated X-ray beam was parallel to the rod axis. The source of radiation was a Coolidge tube with a molybdenum target operated at approximately $28 \mathrm{kv}$. Figure 1 shows the patterns obtained from monocrystalline rods 1 and 2 after different degrees of swaging. Both series of patterns show similar differences in the diffraction spots with increasing degrees of cold-working. However, it is easily seen that the spots in patterns obtained from rod 1 approach an arrangement of threefold symmetry, and those in the patterns obtained from rod 2 approach an arrangement of fourfold symmetry. This indicates that the orientations of the monocrystalline rods 1 and 2 as a whole have changed, so that in the case of rod 1 an octahedral axis is parallel to the rod axis, and in the case of rod 2 a cubic axis is parallel to the rod axis.

The orientation of the crystals after different degrees of swaging was computed from the patterns shown in figure 1 and similar patterns obtained from the crystals in the bicrystalline rods. For patterns of the type shown as 11,12, 21, and 22, figure 1, Greninger's method [2] was used. In this method a specially constructed graph is used to determine the angles between spots or zonal curves on a back-reflection pattern. Then, by comparing a number of these angles with the angles between crystallographic planes, the spots on the pattern can be identified and the orientation determined. However, for patterns such as 13 and 14, which do not show the characteristic zonal curves of the Laue pattern, a slightly different method was required. The latter patterns show the range of orientations (indicated by the length of the circular arcs) of the crystallites around the rod axis. The average orientation of these crystallites in respect to the rod axis is what is required. This can be obtained accurately by constructing a pole figure. However, when the range of orientations is small, as apparently is the case of the crystallites represented by patterns 13 and 14, the average orientation can be determined approximately by the following procedure:

The pairs of arcs in patterns 13 and 14 are diffractions of the $K$ alpha doublet, and, consequently, the corresponding diffracting planes, can be identified. This is conveniently done by making a drawing showing the diffraction rings corresponding to all of the possible planes that could register diffractions of the $\bar{K}$ alpha doublet on a 5 by 7 film at the film-to-specimen distance used in obtaining the diffraction patterns. Then, by placing the film over the drawing, the 
arcs are identified by their coincidence with the rings of the drawing. The centers of the identified arcs then are plotted stereographically, and the angle of rotation [3] is found which brings corresponding poles of a standard projection in best agreement with the plotted poles. This angle of rotation then is used in locating the cubic, octahedral, and dodecahedral poles. The orientations of the crystals in rods 1,2 , 3 , and 4 , before and after different degrees of swaging, are shown in table 2 .

TABLE 2.-Orientations of the crystals in rods 1, 2, 3, and 4 after different degrees of swaging

\begin{tabular}{|c|c|c|c|c|c|c|c|c|c|}
\hline \multirow{2}{*}{$\begin{array}{l}\text { Specimen } \\
\text { number }\end{array}$} & \multirow{2}{*}{$\begin{array}{l}\text { Reduc- } \\
\text { tion in } \\
\text { area }\end{array}$} & \multicolumn{3}{|c|}{$\begin{array}{l}\text { Angles between rod axis } \\
\text { and crystallographic axis }\end{array}$} & \multirow{2}{*}{$\begin{array}{l}\text { Specimen } \\
\text { number }\end{array}$} & \multirow{2}{*}{$\begin{array}{l}\text { Reduc- } \\
\text { tion in } \\
\text { area }\end{array}$} & \multicolumn{3}{|c|}{$\begin{array}{l}\text { Angles between rod axis } \\
\text { and crystallographic axis }\end{array}$} \\
\hline & & Cubic & $\begin{array}{l}\text { Dodeca- } \\
\text { hedral }\end{array}$ & $\begin{array}{l}\text { Octahe- } \\
\text { dral }\end{array}$ & & & Cubic & $\begin{array}{l}\text { Dodeca- } \\
\text { hedral }\end{array}$ & $\begin{array}{l}\text { Octahe- } \\
\text { dral }\end{array}$ \\
\hline $\begin{array}{l}11 . \\
12\end{array}$ & $\begin{array}{r}\text { Percent } \\
0 \\
30\end{array}$ & $\begin{array}{r}\text { Degrees } \\
40 \\
48\end{array}$ & $\begin{array}{r}\text { Degrees } \\
24 \\
30\end{array}$ & $\begin{array}{r}\text { Degrees } \\
16 \\
7\end{array}$ & $\begin{array}{l}31 B^{1}= \\
32 B^{-}\end{array}$ & $\begin{array}{r}\text { Percent } \\
0 \\
30\end{array}$ & $\begin{array}{r}\text { Degrees } \\
40 \\
38\end{array}$ & $\begin{array}{r}\text { Degrees } \\
6 \\
14\end{array}$ & $\begin{array}{r}\text { Degrees } \\
31\end{array}$ \\
\hline 13 & & 50 & 33 & & & & & & \\
\hline 14 & 80 & 55 & 35 & 0 & $\begin{array}{l}41 \mathrm{~A} \\
42 \mathrm{~A}\end{array}$ & $\begin{array}{r}0 \\
30\end{array}$ & $\begin{array}{r}15 \\
9\end{array}$ & $\begin{array}{l}32 \\
36\end{array}$ & $\begin{array}{l}40 \\
47\end{array}$ \\
\hline & $\begin{array}{l}0 \\
30\end{array}$ & $\begin{array}{l}17 \\
10\end{array}$ & $\begin{array}{l}30 \\
36\end{array}$ & $\begin{array}{l}39 \\
45\end{array}$ & $43 A_{--}$ & 60 & 8 & 37 & 48 \\
\hline 23. & 60 & 5 & 41 & $\begin{array}{l}70 \\
50\end{array}$ & $41 B$ & 0 & 49 & 31 & \\
\hline & 80 & 0 & 40 & 00 & $43 \mathrm{~B}$ & $\begin{array}{l}30 \\
60\end{array}$ & $\begin{array}{l}01 \\
54\end{array}$ & $\begin{array}{l}33 \\
34\end{array}$ & \\
\hline 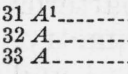 & $\begin{array}{r}0 \\
30 \\
60\end{array}$ & $\begin{array}{r}18 \\
10 \\
6\end{array}$ & $\begin{array}{l}29 \\
36 \\
39\end{array}$ & $\begin{array}{l}38 \\
45 \\
49\end{array}$ & & & & & \\
\hline
\end{tabular}

${ }^{1} A$ and $B$ indicate the two crystals in the bicrystalline rods.

The results show that an 80-percent reduction in area caused an octahedral axis to become parallel to the rod axis in the case of rod 1 and a cubic axis in the case of rod 2. Figure 2 shows the orientations of the rod axis after different degrees of swaging. They lie approximately on a great circle, which indicates that the final orientation resulted from a rotation about one axis. Rod 3 was a bicrystalline rod, crystal $A$ initially having a cubic axis inclined to the rod axis by 18 degrees, and crystal $B$ initially having a dodecahedral axis inclined to the rod axis by 6 degrees. (See specimens $31 A$ and $31 B$, table 2.) The change in orientation of crystal $A$ was similar to that of the single crystal of rod 2. Long diffuse spots made orientation determinations uncertain for crystal $B$ after reductions in area of more than 30 percent. However, the orientations at 30 -percent reduction show that the angle between the dodecahedral axis and the rod axis had increased and the angle between the octahedral axis and the rod axis had decreased. Therefore, the change was similar to that which occurred to the crystal of rod 1. Rod 4 was also a bicrystalline rod, in which crystal $A$ (specimen $41 A$, table 2) had an initial orientation similar to that of the crystal of rod 2, and crystal $B$ (specimen $41 B$, table 2) had an initial orientation similar to that of rod 1 . The changes in orientation resulting from 30 - and 60 -percent reduction in area indicate that swaging produced a tendency for the octahedral axis in crystal $B$ and the cubic axis in crystal $A$ to become parallel to the rod axis. The fact that the changes in orientation of the crystals in the bicrystalline rods were similar to the changes in the monocrystalline rods 
strongly suggests that the changes are independent of crystal boundaries and therefore independent of grain size.

The results, as a whole, indicate that when a copper rod consisting of one or more crystals is deformed by swaging, the crystals rotate until either an octahedral or a cubic axis becomes parallel to the rod

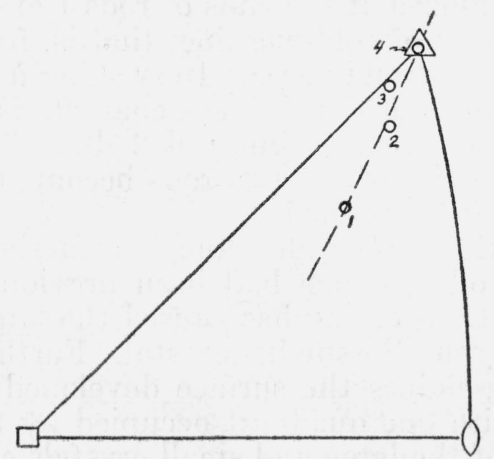

ROD 1

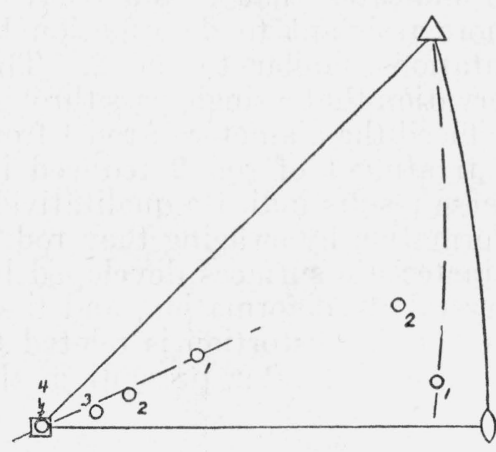

ROD 3

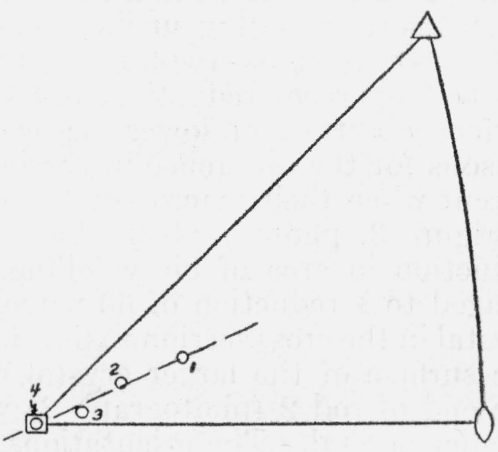

ROD 2

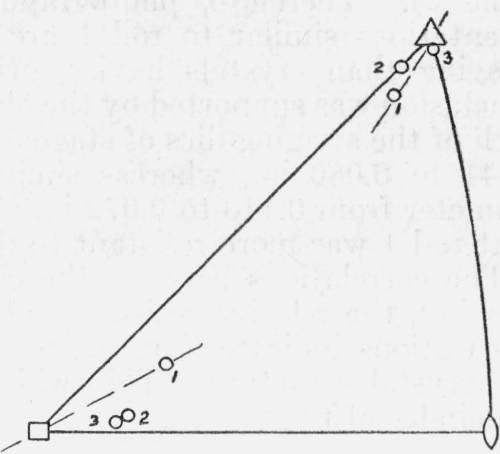

ROD 4

FIGURE 2.-Stereographic projections showing the orientations of the crystals in rods $1,2,3$, and 4 after different degrees of swaging.

The small circles represent the rod axis. The numbers correspond to the identification numbers of table 1.

axis, the choice depending upon which of the two axes initially formed the smallest angle with the rod axis.

\section{APPEARANCE OF THE ENDS OF SWAGED RODS}

By continued swaging of monocrystalline rods 1 and 2, the ends of the rods developed shapes that are related to their ultimate orientations. These characteristic appearances were developed, in each stage of swaging, at the ends of the rods which, before swaging, had been cut off flat and perpendicular to the rod axis. Figure 3, photographs 1 and 2, show these characteristic surfaces developed at the ends of rods 1 and 2 , respectively, in swaging from 60- to 80-percent reduction in area. The depression at the end of rod 1 resembles the inverted corner of a tetrahedron and has a threefold symmetry. It should be 
recalled that the diffraction pattern 14 , figure 1 , which corresponds to this rod and degree of swaging, also has a threefold symmetry. The depression at the end of rod 2 resembles the inverted corner of an octahedron and has a fourfold symmetry. The corresponding diffraction pattern of rod $2(24$, fig. 1$)$ also has a fourfold symmetry. These characteristic surfaces could be recognized at the ends of rods 1 and 2 at stages representing smaller amounts of cold-working, that is, from 0 - to 30 - or from 30- to 60-percent reduction in area. In swaging from 80- to 95-percent reduction, rod 1 did not develop the characteristic surface observed at lower degrees of swaging, but rod 2 did. The reasons for the difference in the behavior of the two rods become apparent when their macrostructures are examined.

Figure 3, photograph 3, shows the surface developed by a slight reduction in area of bicrystalline rod 4 , which had been previously swaged to a reduction of 60 percent. Swaging has caused the larger crystal in the cross section to flow beyond the smaller crystal. Further, the surface of the larger crystal resembles the surface developed at the end of rod 2 (photograph 2) with one quadrant occupied by the smaller crystal. The orientations of the large and small crystals correspond, respectively, to specimens $43 A$ and $43 B$, table 2 . These orientations are approximately the same as rod 2 and rod 1, respectively, after a 60-percent reduction in area (see specimens 23 and 13, table 2). Therefore, photograph 3 indicates that crystals having orientations similar to rod 1 are more resistant to deformation by swaging than crystals having orientations similar to rod 2. This conclusion was supported by the observation that a single pass through each of the swaging dies of stage 5 reduced the diameter of rod 1 from 0.141 to 0.080 in., whereas similar treatment of rod 2 reduced its diameter from 0.140 to 0.072 in. These results indicate qualitatively that rod 1 was more resistant to deformation by swaging than rod 2 .

The correlations between the characteristic surfaces developed by swaging, the relative resistance of crystals to deformation, and their orientations indicate that the manner of the distortion is related to the crystal structure. This will be discussed after presenting the remainder of the results.

\section{METALLOGRAPHIC EXAMINATION}

The macrostructures of cross sections and longitudinal sections of rods 1,2 , and 3 , at various stages of reduction, are shown in figure 4 . The light and dark areas shown in the sections of the monocrystalline rod 1 indicate that the rod contains portions differing in orientation. Similar areas, referred to as deformation bands, have been found in the crystals of rolled copper plate [4] and in compressed specimens of iron that were originally monocrystalline [5]. Inasmuch as these areas indicate differences in orientation, they can be considered as representing sections of crystals, some of which were formed as a result of the deformation.

Diffraction patterns from the central portion of rod 1 have shown a progressive change in orientation. This portion of the rod, therefore, is part of the original crystal, and the other crystals near the periphery have formed from it. In order to distinguish between the original crystal and the new crystals, the former is referred to as a primary crystal and the latter as secondary crystals. 


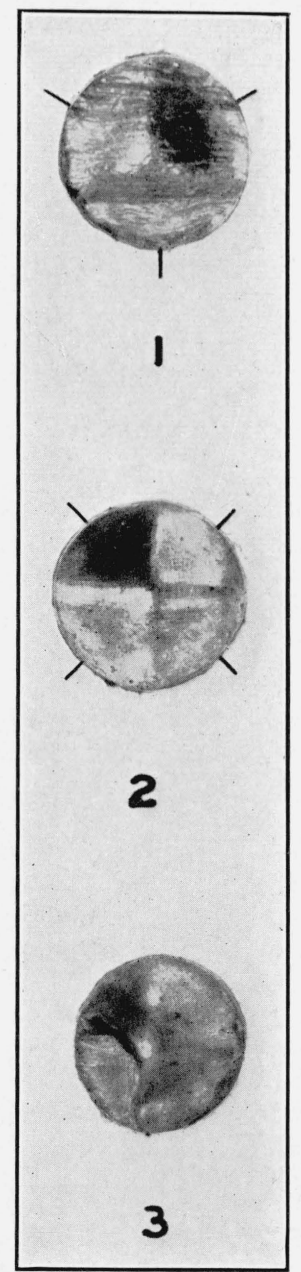

FIGURE 3.-Characteristic surfaces at the ends of the monocrystalline rods 1 and 2 and the bicrystalline rod 4 after swaging.

In the monocrystalline rods the surface metal flowed more than the central metal, thus forming a depression. In the bicrystalline rod the larger crystal flowed more than the smaller. 


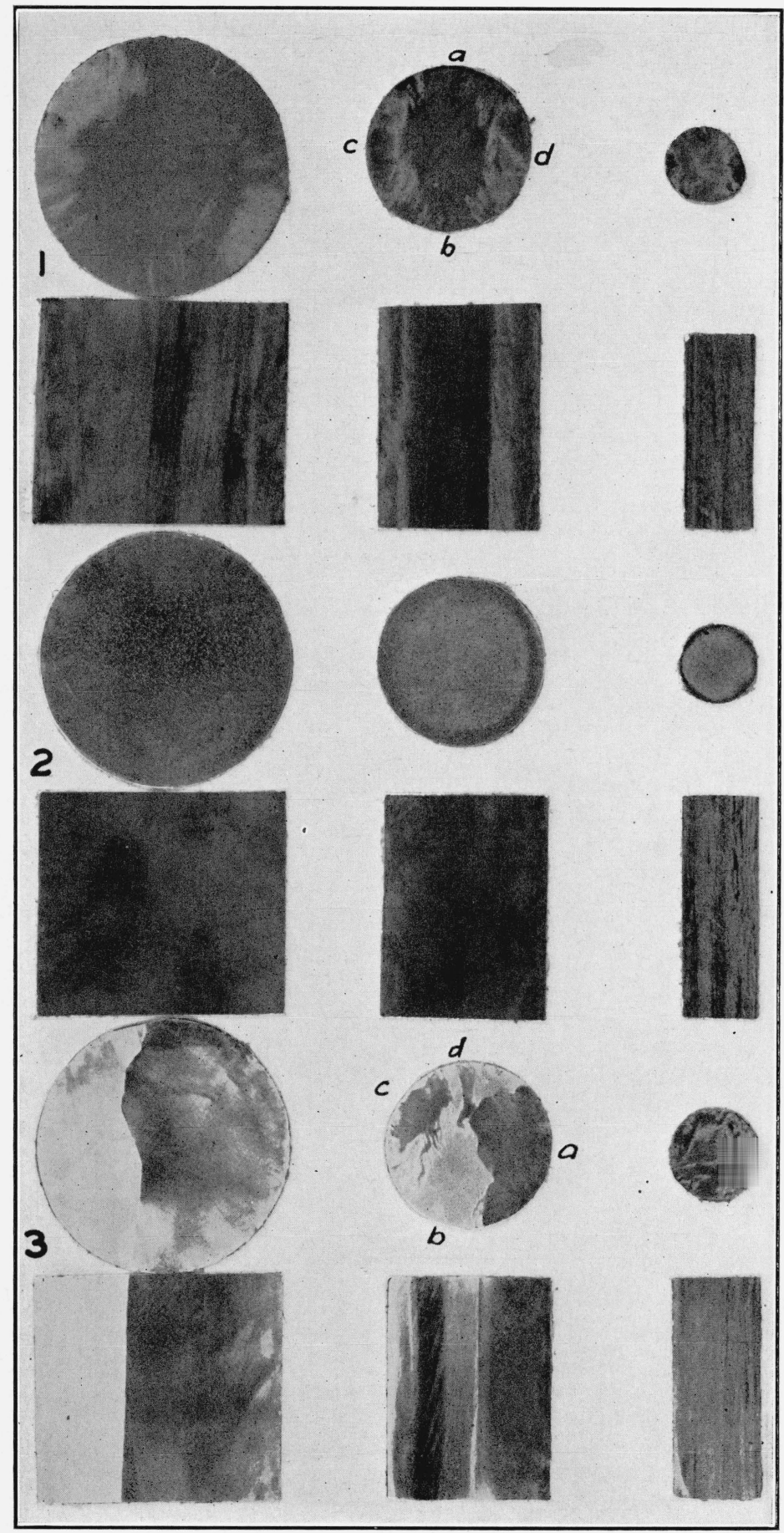

FIGURE 4.-Macrostructure of monocrystalline rods 1 and 2 and bicrystalline rod 3 at different stages in swaging.

Secondary crystals, light and dark areas in No. 1, with different orientations from that of the original crystal, developed during swaging. Rod 2, with different initial orientation from that of rod 1, did not develop secondary crystals during swaging. The crystal at the left of the bicrystalline rod 3 followed the behavior of monocrystalline rod 1, the other crystal resembled monocrystalline rod 2. Specimens etched deeply with 10-percent aqueous ammonium persulfate, $\times 5$, with a ring illuminator. 


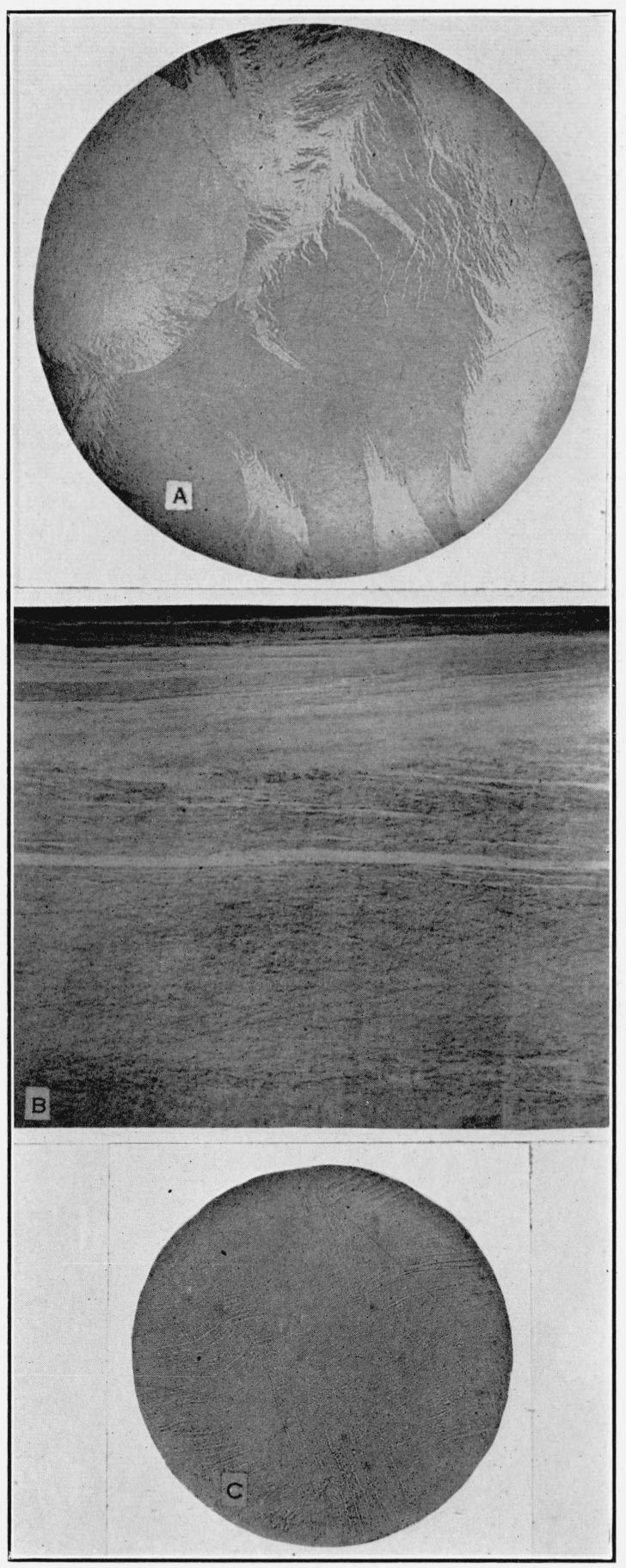

FIGURE 5.-Typical microstructure of copper crystals after swaging to a 95-percent reduction in area.

Etched with 10-percent ammonium persulfate. $A$, cross section of rod $3, \times 40 ; B$, longitudinal section of rod $3, \times 40 ; C$, cross section of $\operatorname{rod} 2, \times 30$. 


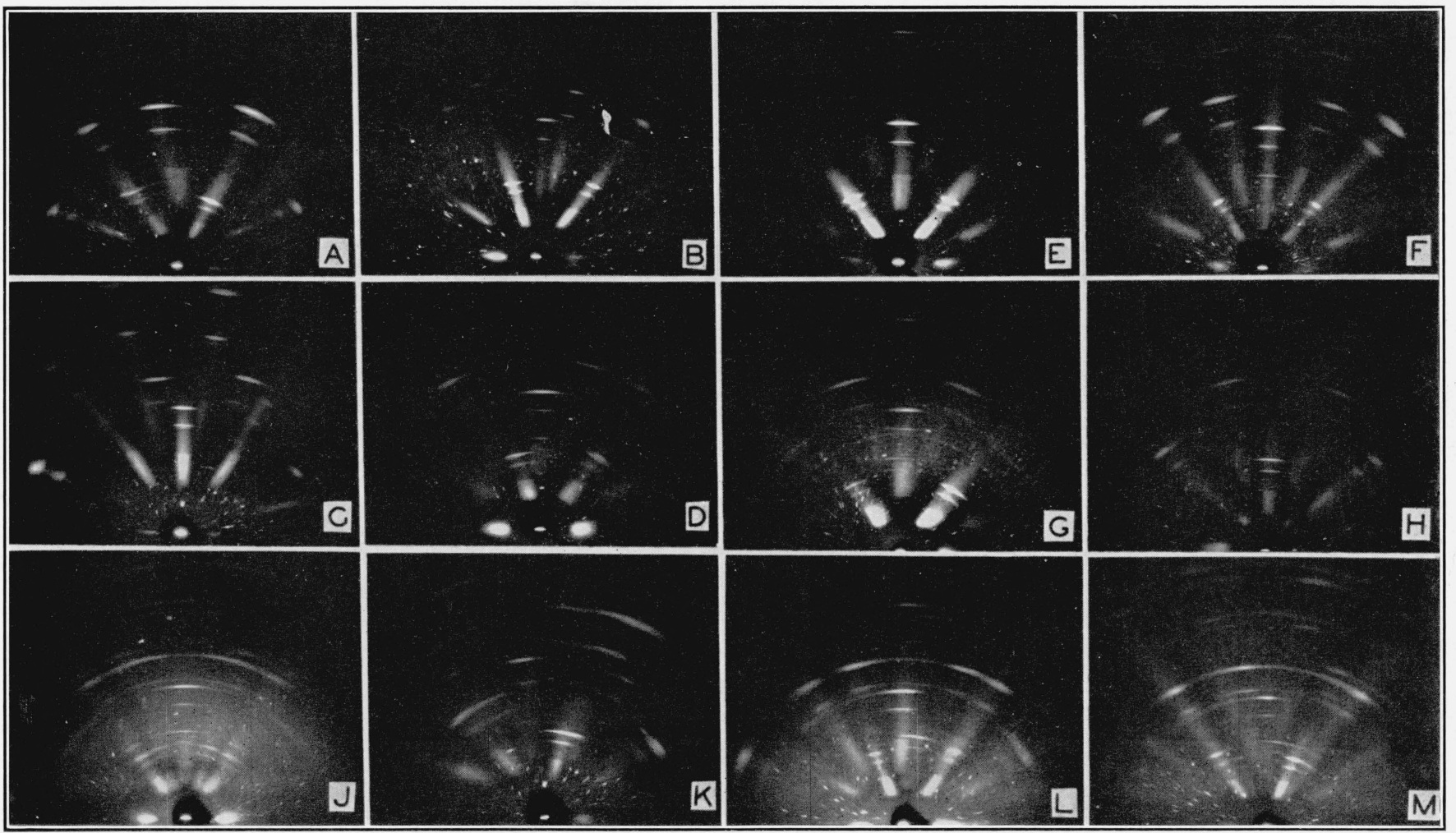

FIGURE 7.-Typical patterns obtained by means of the "reflection" method from monocrystalline and bicrystalline rods of copper swaged to 80and to 95-percent reduction in area. 
The macrostructure of cross sections of the monocrystalline rod 2 after 60 - and 80-percent reductions indicates that it has remained one crystal, and the diffraction patterns 23 and 24, figure 1, indicate that a cubic axis is parallel or nearly so to the rod axis. The orientation of the crystal on the right side of the cross sections of rod 3, figure 4, is approximately the same as the monocrystalline rod 2 after both rods were reduced 60 percent, and like the monocrystalline rod 2, the cross sections of this crystal after 80- and 95-percent reductions show only one crystal. The macrostructure of the crystal on the left side of the cross section, whose orientation after a 30 -percent reduction was similar to the initial orientation of the monocrystalline rod 1, shows primary and secondary crystals after 80 - and 95 -percent reductions, as did the macrostructure of rod 1.

Figure 5 ( $A$ and $B$ ) shows the microstructure of cross and longitudinal sections, respectively, of the bicrystalline rod 3 after 95-percent reduction. The light area in the upper left side of the cross section corresponds to one of the two original crystals, and the remainder of the cross section, containing the jagged crystals, corresponds to the other original crystal. Whenever a microscopic examination of a cross section showed that the original crystal had been converted into several crystals, their boundaries were jagged and irregular, as shown in micrograph $A$, figure 5. It appears that crystals formed during the deformation start at the periphery of the rod and grow in an irregular manner toward the center of the rod. This suggests that the newly formed crystals originate in portions of the rod where the distortion is greatest.

Microscopic examination did not show more than one crystal in the cross sections of the monocrystalline rod 2. However, it did show a heterogeneous structure after 95-percent reduction. This structure is shown in micrograph $C$, figure 5 , and consists of curved narrow bands having two principal directions forming an angle of approximately 90 degrees. Inasmuch as a cubic axis is parallel to the rod axis, this suggests that the curved bands are related to certain planes in the original crystal that have been bent as a result of the deformation.

The presence of a fiber-like structure in the longitudinal section of rod 2 after 95 -percent reduction (fig. 4), the absence of evidence of more than one crystal in the cross section, and the indication of curved planes are important and show that swaging has caused some of the crystallographic planes to bend about axes that are parallel to the rod axis.

The results of the metallographic examination have shown that crystals which rotate so that an octahedral axis tends to become parallel to the rod axis are converted into primary and secondary crystals at a reduction in area of approximately 60 percent. In addition, it has shown that crystals which rotate so that a cubic axis becomes parallel to the rod axis remain one crystal after 95-percent reduction, although the crystallographic planes probably are bent.

\section{ORIENTATIONS OF SECONDARY CRYSTALS}

In order to obtain more definite information on the orientations of the secondary crystals, diffraction patterns were made by the "reflection method" in which the specimen is mounted so that the surface is grazed by the collimated beam of X-rays. The film is usually flat 
and is placed perpendicularly to the collimated beam on the side of the specimen opposite the X-ray tube at a convenient distance $(4.5$ $\mathrm{cm}$ for this work).

The rotational diagram, figure 6 , shows the pattern of diffraction spots obtained by the "reflection method," using monochromatic radiation, when a face-centered cubic crystal is rotated with its axis of rotation perpendicular to the X-ray beam and coinciding with either an octahedral or a cubic axis. In figure 6 the axis of rotation is parallel to the line $A B$. The center represents the undiffracted beam, and the solid and dashed rings (Debye rings) represent the loci of diffractions of the $K$ alpha radiation and the peak of the white

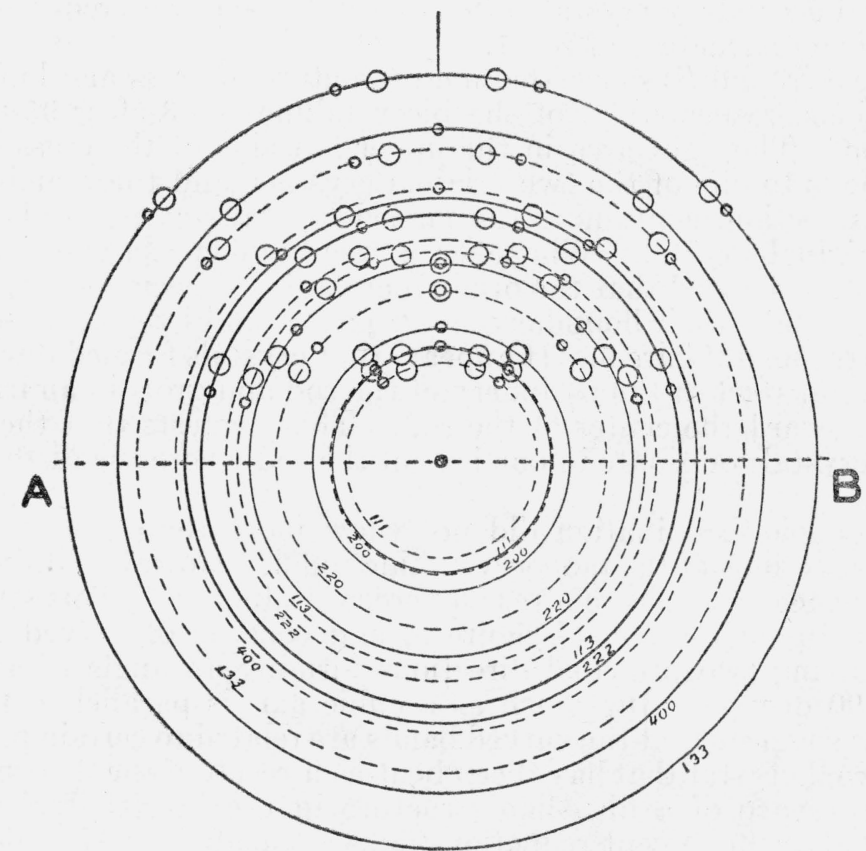

FIGURE 6.-Rotational diagram showing arrangement of diffraction spots from a face-centered cubic crystal.

Large and small circles on the concentric rings represent rotations about an octahedral and a cubic axis, respectively.

radiation, respectively. The large and small circles on the rings show the locations of the diffracted beams from the octahedral and cubic rotations, respectively. The correspondence of the spots on this diagram with the spots on a diffraction pattern obtained from a polycrystalline copper rod, when the rod axis was perpendicular to the X-ray beam, indicates that some of the crystals are oriented with an octahedral axis and others with a cubic axis parallel to the rod axis. The pattern $J$, figure 7 , is a pattern of this type and was obtained from a cold swaged rod of polycrystalline copper. Previous to swaging, this rod was cut from a cold-drawn rod, annealed at $400^{\circ} \mathrm{C}$. for 15 minutes, then swaged to 95-percent reduction in area. Before making the pattern the specimen was deeply etched with diluted nitric acid. This procedure was followed for all subsequent patterns. The method of matching corresponding spots in a diffraction pattern 
with spots in a rotational diagram was used by Schmid and Wassermann [6] when studying the textures of wires. It has been used in this investigation to determine the orientations of crystals in rods 1 , 2 , and 3 after swaging to reductions in area of 80 and 95 percent.

The patterns shown in figure 7 , with the exception of $J$, are typical of the patterns obtained from rods 1, 2, and 3 after 80 - and 95 -percent reductions in area. The patterns $A, B, C$, and $D$ contain spots corresponding only to the large circles of the rotational diagram, figure 6 . This indicates that the crystals irradiated by the primary beam are oriented with an octahedral axis parallel to the rod axis. It can be seen also that some of the large circles in the rotational diagram are not represented in the diffraction pattern. This indicates that the crystals, in addition to having an octahedral axis parallel to the rod axis, have other axes that approximately are oriented in respect to a direction perpendicular to the rod axis. The orientations of these crystals would be equivalent to a rotation, of less than 120 degrees, of a single crystal about an octahedral axis which is parallel to the rod axis. Rotation of 120 degrees about an octahedral axis would reproduce the orientation of the crystal. The orientations represented by patterns $A, B, C$, and $D$ are referred to as type $A$. The patterns $E, F, G$, and $H$ contain spots corresponding only to the small circles of the rotational diagram and, like the patterns $A, B, C$, and $D$, some of the small circles are not represented by spots in the diffraction patterns. The orientations of the crystals represented by the patterns $E, F, G$, and $H$ therefore are equivalent to a rotation, of less than 90 degrees, of a single crystal about a cubic axis which is parallel to the rod axis. A rotation of 90 degrees about a cubic axis would reproduce the orientation of the crystal. The orientations represented by patterns $E, F, G$, and $H$ are referred to as type $B$. The pattern $\bar{K}$ represents orientations intermediate to types $A$ and $B$ and is referred to as type $C$.' This particular pattern indicates orientations such that a cubic axis of the crystals is inclined to the rod axis by about 15 degrees. The patterns $L$ and $M$ indicate that the beam of $\mathrm{X}$-rays irradiated crystals having orientations corresponding to types $A$ and $C$.

The orientations of crystals that would produce patterns of the types $A$ and $B$ are shown in the projections of figure 8. Projection $A$ corresponds to type $A$ with an angle of rotation of about 40 degrees ${ }^{2}$ about the rod axis. This value is obtained from the arc length of the spots in pattern 14, figure 1. The angle of rotation about axes perpendicular to the rod axis is 15 degrees and is the average value of the arc length of the spots shown in the patterns of figure 7. Projection $B$ corresponds to type $B$ and shows the angle of rotation about the rod axis to be 30 degrees. This value represents the arc length of the spots in pattern 24, figure 1 . The angle of rotation about axes perpendicular to the rod is 15 degrees, the same as projection $A$. It is not to be implied that orientations of types $A$ and $B$ have definite angles of rotation such as are indicated by projections $A$ and $B$. The projections represent in a general way the range of orientations that could produce patterns of the type shown in patterns $A$ to $H$, figure 7 . Actually, the range of orientations probably would show a larger angle of rotation around the rod axis as the distance between the

3 The angle of rotation around the rod axis is equivalent to the arc length of the areas containing the poles, and the rotation about an axis perpendicular to the rod axis is equivalent to the are width of the areas c ontaining the poles. 

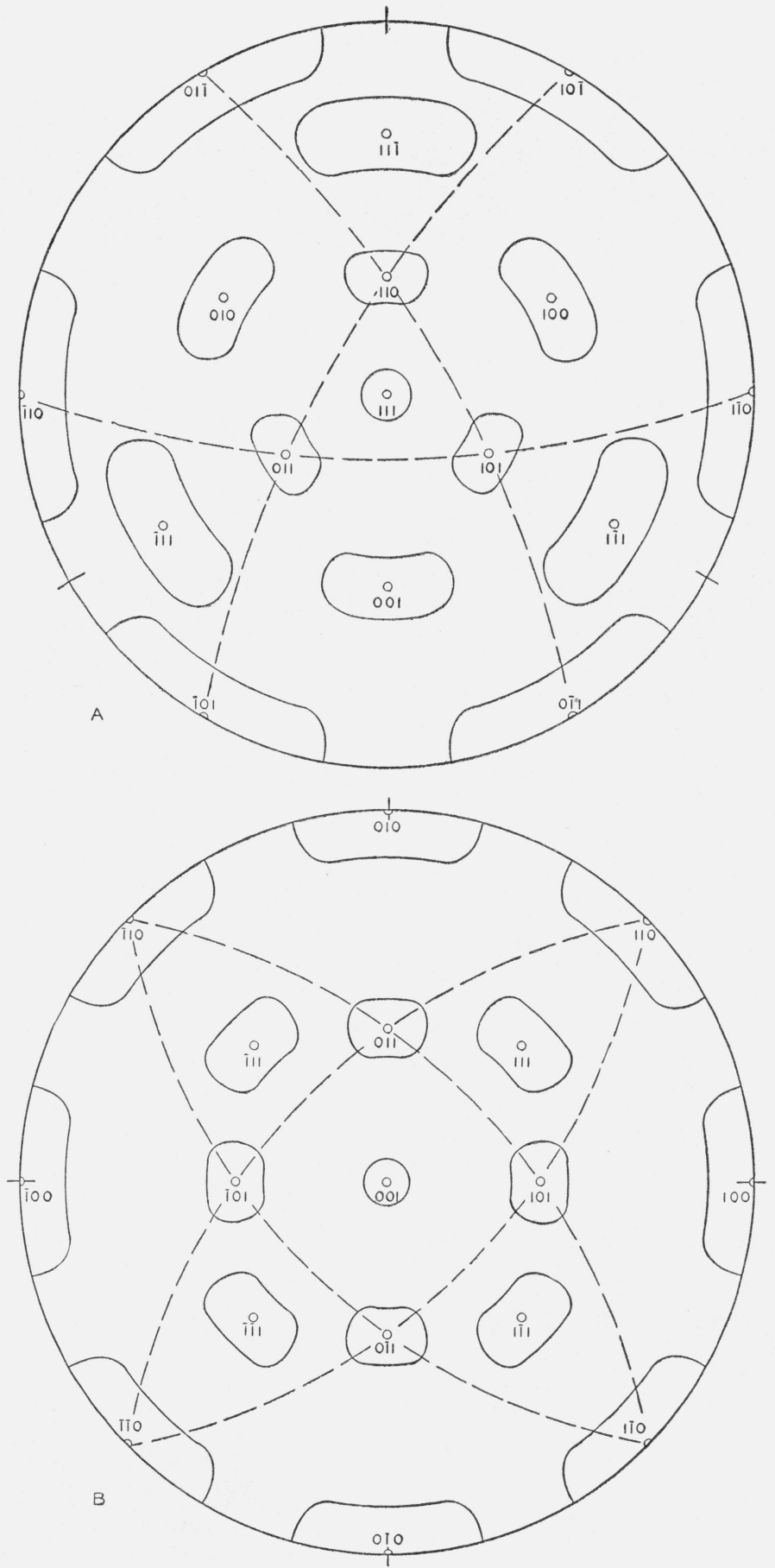

Figure 8.-Stereographic projections showing the octahedral orientation, $A$, and the cubic orientation, $B$.

The rod axis is normal to the projection planes. 
center of the rod and the area examined increases. The angle of rotation around the rod axis would increase also as the percentage reduction in area increases.

The macrostructure of a cross section of rod 1 after an 80-percent reduction, figure 4 , shows that the primary crystal extends to the periphery at $a$ and $b$. Diffraction patterns obtained from areas on the surface of the rod corresponding to $a$ and $b$ indicated orientations of type $A$. It may be confusing to imply that a crystal seen in the macrostructure has more than one orientation, but this means simply that a macroscopic crystal consists of crystallites with a definite range of orientations, as revealed by diffraction patterns. In order to avoid confusion, the apparent crystal seen in the macrostructure is referred to as a crystal, and its orientation is the average orientation of the crystallites of which it consists. In the case of crystals having orientation ranges of the types $A$ and $B$, the average orientation is respectively an octahedral axis and a cubic axis parallel to the rod axis; see projections $A$ and $B$, figure 8. Diffraction patterns representing areas corresponding to the secondary crystals of rod 1 , shown as $c$ and $d$ in figure 4, indicated orientations of the type $C$. The experimental set-up and the smallness of areas corresponding to $a, b, c$, and $d$ did not permit a similar correlation of diffraction patterns with macrostructure after 95-percent reduction. However, numerous patterns made with the specimens rotated slightly about the rod axis after each exposure indicated orientations of types $A$ and $B$, although some patterns were still a mixture of types $A$ and $C$. These results indicate that swaging secondary crystals having orientations of the type $C$ changes the orientation into type $B$.

Patterns obtained from rod 2 after 80 - and 95-percent reductions indicated orientations only of type $B$. This might be expected as the macro-structure of the cross sections at these reductions, figure 3 , did not show any secondary crystals. Patterns obtained from areas on the periphery of the rod corresponding to mark $a$, figure 4 , of the bicrystalline rod 3 after an 80-percent reduction indicated orientations of the type $B$. It has been pointed out that this crystal has an orientation similar to the monocrystalline rod 2 , and also that it did not show any secondary crystals. Patterns obtained from areas corresponding to $b$ indicated a mixture of types $A$ and $C$. Areas corresponding to $c$ and $d$ indicated types $A$ and $C$, respectively. Numerous patterns of this rod after a 95-percent reduction, with the specimens rotated slightly after each exposure, indicated orientations of types $A$ and $B$. These results indicate that the crystal of the bicrystalline rod which developed secondary crystals was similar to the crystal of rod 1 .

The results as a whole indicate that crystals initially having a cubic axis approximately parallel to the rod axis are "stable" during deformation by swaging, whereas crystals whose orientation is changing, so that an octahedral axis tends to become parallel to the rod axis, are unstable. These "unstable" crystals develop secondary crystals with orientations of type $C$, that is, intermediate between having an octahedral axis or a cubic axis parallel to the rod axis. Further distortion by swaging changes the initial orientation of the secondary crystal to an orientation consisting of a cubic axis parallel to the rod axis. The ultimate state, therefore, of a crystal having an initial orientation such that an octahedral axis tends to align itself with the rod axis is a mixture of crystals, each having an octahedral axis or a cubic axis parallel to the rod axis. 


\section{DISCUSSION}

\section{MANNER OF CHANGING THE ORIENTATION OF A COPPER CRYSTAL BY SWAGING}

It is generally conceded that metallic crystals can be distorted plastically only by slipping on certain crystallographic planes and in certain crystallographic directions. Investigations $[7,8]$ have shown that when a distortion by tensile or compressive stress occurs in this manner, the crystal changes its initial orientation to an orientation which is stable to a further distortion. In the case of face-centered cubic crystals, such as copper, the distortion takes place on octahedral planes and in dodecahedral directions. There are four octahedral planes in a cubic crystal and three dodecahedral directions in each octahedral plane, and, therefore, there are 12 possible slip systems.

The first stage in the distortion of a copper crystal by a tensile stress involves slip in the system having the greatest resolved shear stress. As the distortion proceeds, the orientation of the crystal changes in a way that is equivalent to rotating the direction of slip so that it tends to become parallel to the tensile stress. This rotation will in time bring the crystal to a position where another slip system has the same resolved shear stress as the active system. The second stage of the distortion then proceeds by an equal amount of slip on each of the active slip planes, until the two active slip directions and the tensile stress direction lie in the same plane, that is, until the icositetrahedral direction [112] coincides with the tensile-stress direction. The change in orientation accompanying the second stage is equivalent to a rotation about a dodecahedral axis. Further distortion does not change this orientation. It is evident, then, that a copper crystal distorted by a tensile stress changes its initial orientation to a stable orientation in a manner equivalent to rotation about two axes.

The distortion of a copper crystal under compressive stress also involves two stages. The first stage is accomplished by slip in the system having the greatest resolved shear stress, and by a change in orientation that is equivalent to a rotation causing a normal to the active slip plane to become more parallel to the compressive stress. The rotation continues until another slip system has the same resolved shear stress as the active system. The distortion then commences the second stage, which is accomplished by two slip systems. The change in orientation during the second stage is equivalent to a rotation about a cubic axis, until the normals of the two active slip planes and the compressive stress direction are in the same plane. In this position a dodecahedral axis coincides with the compressive stress direction. A further distortion does not change this orientation, and, like the distortion by a tensile stress, it was attained in a manner equivalent to rotation about two axes.

The changes in orientation of a copper crystal resulting from a distortion by swaging are accomplished in a somewhat different manner from those resulting from simple tensile or compressive stress. It has been pointed out that the orientations of a copper crystal after different degrees of swaging lie on a great circle when plotted stereographically, as in figure 2. This means that the two stable orientations were attained in a manner equivalent to a rotation about one axis and indicates that the number of active slip systems operating 
at the beginning of the distortion was the same as the number operating at the stable orientation, although the degree of activity of the different systems varied until the stable orientation was attained. The two stable orientations attained by swaging are shown in figure 8. The locations of the octahedral planes are shown as great circles (dashed); the dodecahedral directions are at the intersections of the octahedral planes. ${ }^{3}$ It is evident that the octahedral planes and dodecahedral directions are symmetrically placed around the rod axis in both of these orientations. It is possible then that these stable orientations were attained by six slip systems symmetrically placed around an octahedral axis in the case of the octahedral orientation, figure $8 A$, and eight slip systems symmetrically placed around a cubic axis in the case of the cubic orientation, figure $8(B)$.

The attainment of the two stable orientations by six and eight slip systems becomes more probable when they are correlated with the characteristic surfaces developed at the ends of the swaged monocrystalline rods 1 and 2 . The short lines shown on the photographs 1 and 2 of figure 3 correspond to the short lines crossing the circumferences of the projections $A$ and $B$, figure 8, respectively. It can be seen that the surfaces shown in figure 3 could be formed by slip in six slip systems in the case of the octahedral orientation and eight in the case of the cubic orientation. The fact that surfaces similar to these could be produced before the stable orientation was attained supports the contention that these slip systems were operating from the beginning of the distortion.

It is noteworthy that the arrangements of slip systems in the octahedral and cubic orientations have, respectively, a threefold and a fourfold symmetry to the direction of flow. This suggests that the direction of flow that is required by the deformation might be the factor determining the selection of active slip systems. If this be true, then the stable orientations of copper attained by swaging should be attained regardless of the kind of slip plane or slip direction, because any family of planes or directions has a threefold and fourfold arrangement about an octahedral axis and a cubic axis, respectively. Recent experiments by Barrett [5] appear to support this contention. In his experiments, monocrystalline disks of iron were compressed in such a way that the disc retained its circular shape throughout the compression. This manner of deforming a disk is similar to the deformation of a rod by swaging. In the case of the compressed disc the diameter is increased, and in the case of the swaged rod the diameter is decreased. The body-centered cubic crystals of alpha iron are known to distort by slipping on the dodecahedral (110), icositetrahedral (112), and the hexakis octahedral (123) planes and in an octahedral direction [111]. Barrett's results showed the same stable orientations for bodycentered iron as are attained for face-centered crystals of copper, that is, an octahedral axis or a cubic axis parallel to the cylindrical axis of the specimen.

From this discussion the conclusion is drawn that, if the distortion of a crystal produces a cylindrical shape, it would be accomplished by slip systems symmetrical to an octahedral or a cubic axis, and this axis, on continued distortion, would become parallel to the rod axis. In the case of copper there are six slip systems symmetrical to an

\footnotetext{
${ }^{3}$ In the cubic system crystallographic directions are normal to their corresponding planes, that is, the cubic direction [100] is normal to the cubic plane (100), the dodecahedral direction [110] is normal to the dodecahedral plane (110), the octahedral direction [111] is normal to the octahedral plane (111), etc.
} 
octahedral axis and eight slip systems symmetrical to a cubic axis. The results indicate that the crystals rotate until either an octahedral or a cubic axis becomes parallel to the rod axis, the choice depending upon which of the two axes initially formed the smallest angle with the rod axis. It is pointed out in a subsequent section of this paper that this conclusion does not hold when monocrystalline rods of iron are distorted by swaging.

\section{BENDING OF SLIP PLANES AND A POSSIBLE ORIGIN OF SECOND- ARY CRYSTALS}

Deformation by swaging produces a rod which is uniform in diameter, that is, the rod is a perfect circular cylinder. Therefore, in coldworking a monocrystalline rod by swaging, the distorted crystal is a perfect cylinder at all degrees of swaging. Obviously this shape cannot be produced perfectly, and the slip planes remain flat when there are a limited number of slip systems, as discussed in the preceding section. It is reasonable then to conclude that the slip planes which are initially flat become curved during swaging, and that the later stages are accomplished by curved surfaces slipping over one another. This kind of distortion has been called "bend gliding" (Biegegleitung) [8] and has been used to explain the asterism shown in Laue patterns of distorted crystals. Briefly the explanation is that during a distortion the metal between the slip planes is bent over a curvature sufficient to strain some of the interatomic bonds beyond their elastic limit, thereby causing the metal to break into blocks, called crystallites, having approximately the same orientation and each being a perfect crystal. Diffractions from the crystallites produce the asterism of the Laue patterns. If the distortion has been severe, the range of orientations of the crystallites is too large to produce the characteristic radial asterism but does produce a pattern consisting of spots elongated peripherally and arranged on concentric circles, as is shown in figure 7. From this explanation it is seen that the range of orientations of the crystallites is a measure of the amount of bending which the slip planes have undergone. The ranges of orientations of the crystallites in crystals distorted by an amount of swaging equivalent to a reduction in area of 80 to 95 percent have been shown in figure 8 . The dimensions of the ranges indicate that the slip planes are curved more around an axis parallel to the rod axis than around an axis perpendicular to the rod axis. The results of the experiments with swaged crystals of copper therefore appear to be consistent with the conclusion that a distortion can be accomplished by the slipping of curved sheets of crystallites over one another.

Heretofore, the shape of the crystallites has not been considered. The shape becomes important when it is remembered that the sheets themselves as well as the directions of their movement must be related to certain crystallographic planes and directions. It seems that this condition can be satisfied, at least for the distortion of copper crystals by swaging, if the assumption is made that certain octahedral planes, spaced equally at a distance of the order of $10^{-5} \mathrm{~cm}$ are potential slip planes. Then a distortion sufficiently severe to produce slip on all of the potential slip planes parallel to the three octahedral planes that are symmetrical to an octahedral axis would divide the crystal into crystallites having a rhombohedral shape. The faces of a rhombohedron in this case would be octahedral planes and the 
edges would be dodecahedral directions. It is more difficult to shear these unit rhombohedra than it is for them to slide over one another. Consequently, it follows that the more out-of-line they become the more necessary it would be for them to move in directions parallel to their edges.

However, it is interesting to note that each unit rhombohedron would contain two potential slip planes, and that, if slip should occur on these planes, the unit rhombohedron would be divided into two tetrahedra and one octahedron. These potential slip planes represent the only means of destroying the unit rhombohedron. Therefore, these planes must be considered a source of weakness for the unit rhombohedron. This gives a possible explanation for the origin of secondary crystals such as developed during the swaging of monocrystalline rod 1 . It is quite possible that during swaging a unit rhombohedron would be rotated to a position where the stresses would be sufficient to cause a shear along the two potential slip planes. The results indicate that this would occur when the angle between a cubic axis of the rhombohedron and the rod axis was approximately 15 degrees. After a number of adjacent rhombohedra had been sheared this portion would begin to slip over the surfaces of the newly formed tetrahedra and octahedra. This kind of a distortion would be equivalent to a distortion by eight slip systems symmetrical to a cubic axis.

It is concluded, therefore, that crystals can be distorted by slipping over curved surfaces composed of crystallites whose surfaces and edges are the slip planes and slip directions in the undistorted crystal. This property gives the crystal a limited amount of plasticity in all directions, similar to the plasticity of amorphous substances, and at the same time permits the distorted crystal to retain the characteristics of a single crystal.

\section{FIBROUS TEXTURE OF COLD-WORKED RODS}

The structural changes taking place in the crystals of the bicrystalline rods are good examples of crystals behaving according to the conclusions stated in the preceding paragraphs. The flow of metal in each crystal was similar to the flow of metal in the rod as a whole, that is, the cross section was uniformly decreased as the length was increased, and the results showed that the orientation of each crystal was changed progressively to a stable orientation after diffraction patterns had indicated the crystals to be composed of crystallites. From this it is concluded that each crystal in a polycrystalline rod of copper behaves as one of the crystals in the bicrystalline rods. There is, however, a difference in respect to secondary crystals. So far as can be ascertained, secondary crystals are not found in the fiber-like crystals of cold-worked rods of polycrystalline copper.

The crystals of a polycrystalline rod of copper, cold-worked 60 percent or more, would be rotated to either an octahedral or a cubic orientation. If the crystals had been oriented randomly and had not developed secondary crystals, the ratio of the fiber-like crystals having the octahedral orientation to those having the cubic orientation would be equal to 1. Results of Schmid and Wassermann [6] show that this ratio is 1.5. The discrepancy in the two values might be explained on the basis that the material used by Schmid and Wassermann, in their wire-drawing experiments, had a slight degree of 
preferred orientation, favoring the stable octahedral orientation, as a result of its previous treatment. It is known that the preferred orientation developed in cold-rolled plates of copper is not completely removed by annealing.

There are apparent exceptions to the conclusion that an octahedral or a cubic orientation is attained when the shape of the crystal produced during the distortion is a cylinder. It has been reported that cold-worked rods of aluminum have only the octahedral orientation[6]. Aluminum is a face-centered crystal; therefore, the slip planes and slip directions are the same as copper. These reported results indicate that a monocrystalline rod of aluminum having a cubic axis parallel to the rod axis would rotate to the octahedral orientation during swaging or would develop secondary crystals that would do likewise. This is not consistent with the results obtained from swaged crystals of copper.

Another exception is found in the work of Barrett [9], who showed that iron crystals of different orientations when swaged or drawn immediately developed numerous secondary crystals. The secondary crystals rotated away from their initial orientation, eventually reaching a stable orientation when a dodecahedral axis became parallel to the rod axis. The resulting state of the original crystal after a 90percent reduction in area consisted of numerous secondary crystals having a dodecahedral axis parallel to the rod axis, and other axes randomly oriented in respect to a direction perpendicular to the rod axis. From these results, it was concluded that all of the crystals in polycrystalline rods behave in a similar manner, thus accounting for the fibrous texture found in iron rods. The present investigation indicates that the fibrous texture of iron rods differs from that of copper rods in respect to secondary crystals and to stable orientations. In iron rods the original crystals are converted into numerous secondary crystals that rotate to a dodecahedral orientation, whereas in copper rods the original crystals are rotated either to an octahedral or a cubic orientation.

The conclusion, that if the distorted shape was cylindrical, the active slip systems would be symmetrical to an octahedral axis or a cubic axis, which eventually becomes parallel to the cylinder axis, obviously is not correct for iron crystals that are swaged or drawn, although it appears to be correct for iron crystals that are deformed plastically by compression and for copper crystals that are swaged. There is a possible explanation for this apparent inconsistency. In swaging or drawing, the flow of metal is parallel to the rod axis, whereas in compressing a disk the flow of metal is perpendicular to the axis of the disk. If a monocrystalline disk of iron having either an octahedral or a cubic orientation (octahedral or cubic axis parallel to axis of the disk) is compressed, the angles between the direction of flow and the directions of slip are 19.5 and 35 degrees, respectively, whereas if a monocrystalline iron rod having either of these orientations is swaged or drawn, the angles between the direction of flow and the slip directions are 70.5 and 55 degrees, respectively. It seems reasonable then that if the angles between the direction of flow and directions of slip were such as to permit easy flow in compression that in swaging or drawing these angles would be too large for easy flow. The iron crystal, therefore, begins to develop secondary crystals having an easier flow. Crystals of this type are apparently those having a dodec- 
ahedral axis parallel to the rod axis. The angle between the directions of slip and flow in this case is 35 degrees. In swaging a copper crystal the angle between the directions of slip and of flow is 35 degrees when the crystal has the octahedral orientation and 45 degrees when the orientation is cubic. In either case an easy flow of material occurs without change in orientation. However, when the orientation is dodecahedral, the angle between the directions of slip and of flow is 60 degrees, which is too large for easy flow without change in orientation. The crystal tends to change its orientation from dodecahedral to octahedral with certain portions forming secondary crystals which rotate toward the cubic orientation.

\section{SUMMARY}

Two monocrystalline and two bicrystalline rods of copper were swaged through the same dies to a reduction in area of approximately 95 percent. Small pieces were cut from the swaged rods after 30-, 60-, 80-, and 95-percent reduction in area. The structures of these pieces, as well as the initial structures, were examined by $\mathrm{X}$-ray diffraction and metallographic methods.

In one monocrystalline rod the octahedral axis that was initially inclined to the rod axis by 16 degrees became parallel to the rod axis as a result of swaging, whereas in the other monocrystalline rod, the cubic axis that was initially inclined to the rod axis by 17 degrees became parallel to the rod axis. These apparently stable orientations were aiso obtained for crystals in the bicrystalline rods when their initial orientations were similar to the initial orientations of the monocrystalline rods. The changes in orientation with different degrees of swaging were equivalent to a rotation of the crystal about one axis. The crystals that rotated to the octahedral orientation developed secondary crystals that rotated to the cubic orientation. The crystals that rotated to the cubic orientation did not develop secondary crystals. Diffraction patterns and the appearance of the ends of the swaged rods indicated that the crystals had been distorted by slipping over curved surfaces and in directions related to the octahedral planes and dodecahedral directions of the undistorted crystal.

The results indicated that the rotation of the crystal to the octahedral orientation was accomplished by the operation of six slip systems symmetrical to the octahedral axis that became parallel to the rod axis, and in a similar manner, the cubic orientation was attained by the operation of eight slip systems symmetrical to the cubic axis that became parallel to the rod axis. Whether the crystal assumes the octahedral or the cubic orientation depends upon which axis initially formed the smaller angle with the rod axis.

\section{REFERENCES}

[1] P. W. Bridgman, Certain physical properties of single crystals of tungsten, antimony, bismuth, tellurium, cadmium, zinc, and tin, Proc. Am. Acad. Arts Sci. 60, 307-314 (1925).

[2] Alden B. Greninger, A back-reflection Laue method for determining crystal orientation, Z. Krist. [A] 90, 424-432 (1935); Trans. Am. Inst. Mining Met. Engrs. 11\%, 61-74 (1935).

[3] Charles S. Barrett, The stereographic projection, Trans. Am. Inst. Mining Met. Engrs. 124, 29-59 (1937). 
[4] F. Johnson, Influence of cold-rolling upon mechanical properties of oxygen-free copper, J. Inst. Metals 21, 335-346 (1919).

[5] Charles S. Barrett, The structure of iron after compression, Metals Tech. 5, (1938); Am. Inst. Mining Met. Engrs. Tech. Pap. 977.

[6] E. Schmid and G. Wassermann, The texture of cold-drawn wire, Z. Physik 42, 779-794 (1927).

[7] E. Schmid and W. Boas, Crystal Plasticity (J. Springer, Berlin, 1935).

[8] C. F. Elam, Distortion of Metal Crystals (Oxford Press, Cambridge, London, 1935).

[9] Charles S. Barrett and L. H. Levenson, Structure of iron after drawing, swaging, and elongating in tension, Metals Tech. 6, (1939); Am. Inst. Mining Met. Engrs. Tech. Pap. 1038.

Washington, February 28, 1939. 